\title{
Correlación fenotípica y estimación del peso vivo en bovinos criollos
}

\author{
Phenotypic correlation and estimation of bodyweight in creole cattle
}

José L. Contreras P. ${ }^{1,5}$, Alfonso Cordero F. ${ }^{2}$, Blas Reymundo C. ${ }^{3}$, Hebert E. Ramos A. ${ }^{3}$, James Curasma C. ${ }^{1}$, Alfredo Delgado C. ${ }^{4}$

\section{Resumen}

\begin{abstract}
El objetivo del estudio fue determinar las correlaciones fenotípicas entre el peso vivo (PV) y las medidas biométricas de 90 bovinos criollos, de ambos sexos y edades diversas, procedentes de la Comunidad de Conayca en Huancavelica, Perú, para definir ecuaciones de predicción del PV. Se hicieron mediciones de ancho de cabeza (AC), longitud de cabeza (LC), ancho de tórax (ATO), altura de tórax (ALT), longitud de cuerpo (LCU), perímetro torácico (PT), altura de cruz (ACR), ancho de grupa (AGR), longitud de grupa (LGR), perímetro de caña anterior (PCA), distancia dorso lumbar (DDL), altura de grupa (ALG), longitud de tórax (LTO), largo de cuerpo (LAC) y perímetro abdominal (PAB). Las observaciones fueron analizadas utilizando modelos lineales, incluyendo el efecto del sexo y la edad dentaria de los animales. Las medidas biométricas fueron ajustadas en una regresión lineal múltiple de primer grado para obtener las ecuaciones de predicción de PV de los animales. La edad dentaria fue la fuente de variación más importante en cada una de las características, excepto para ALT. El sexo solamente afectó significativamente al PCA y ALG. Se detectaron correlaciones fenotípicas altas y significativas $(\mathrm{p}<0.001)$ entre el PV y las medidas de PT (0.91), LC (0.79), ACR (0.77), ALG (0.78) y LAC (0.89). El PT + $\mathrm{ACR}+\mathrm{LAC}$ presentó la ecuación de predicción más precisa $\left(\mathrm{R}^{2}=1.0000\right)$, seguido por la participación del PT + LAC $\left(\mathrm{R}^{2}=0.9775\right)$ y del PT $\left(\mathrm{R}^{2}=0.9274\right)$. Se concluye que el PV de los bovinos criollos estudiados se puede predecir satisfactoriamente a partir del PT, o PT $+\mathrm{LAC}$ o PT + ACR + LAC, donde la edad dentaria es el factor que influye en mayor grado en las predicciones.
\end{abstract}

Palabras clave: peso vivo, bovinos criollos, interrelaciones productivas, biometría

${ }^{1}$ Laboratorio de Nutrición Animal y Evaluación de Alimentos, Universidad Nacional de Huancavelica, Perú

${ }^{2}$ Universidad Nacional de Huancavelica, Perú

${ }^{3}$ Laboratorio de Anatomía y Fisiología Animal, Universidad Nacional de Huancavelica, Perú

${ }^{4}$ Clínica de Animales Mayores, Facultad de Medicina Veterinaria, Universidad Nacional Mayor de San Marcos, Lima, Perú

${ }^{5}$ E-mail: jose.contreras@unh.edu.pe

Recibido: 20 de marzo de 2019

Aceptado para publicación: 20 de diciembre de 2019

Publicado: 31 de marzo de 2020 
The aim of this study was to determine the phenotypic correlations between bodyweight (PV) and the biometric measurements of 90 Creole cattle, of both sexes and diverse ages, from the Conayca Community in Huancavelica, Peru, to define prediction equations of PV. Head width (AC), head length (LC), chest width (ATO), chest height (ALT), body length (LCU), chest perimeter (PT), height at the withers (ACR), rump width (AGR), rump length (LGR), anterior fetlock perimeter (PCA), lumbar back distance (DDL), rump height (ALG), chest length (LTO), body length (LAC) and abdominal perimeter $(\mathrm{PAB})$ were measured. The observations were analysed using linear models, including the effect of sex and age based on teeth growth. The biometric measurements were adjusted in a first order multiple linear regression to obtain the prediction equations of PV of the animals. Dental age was the most important source of variation in each of the characteristics, except for ALT. Sex only significantly affected PCA and ALG. High and significant phenotypic correlations $(\mathrm{p}<0.001)$ were detected between the $\mathrm{PV}$ and the measurements of PT (0.91), LC (0.79), ACR (0.77), ALG (0.78) and LAC (0.89). The PT + $\mathrm{ACR}+\mathrm{LAC}$ presented the most accurate prediction equation $\left(\mathrm{R}^{2}=1.0000\right)$, followed by the PT + LAC $\left(\mathrm{R}^{2}=0.9775\right)$ and the PT $\left(\mathrm{R}^{2}=0.9274\right)$. It is concluded that the PV of the Creole cattle studied can be satisfactorily predicted from the PT, or PT + LAC or PT + $\mathrm{ACR}+\mathrm{LAC}$, where dental age is the factor that most influences predictions.

Key words: body weight, native cattle, productive interrelations, biometry

\section{INTRODUCCIÓN}

La sierra peruana alberga el $78.8 \%$ de la población de bovinos del Perú (Caballero y Flores, 2006). La ganadería en esta región está constituida por una fauna autóctona (camélidos sudamericanos) así como por otra basada en animales introducidos por los españoles de la península Ibérica e Islas Canarias en el siglo XVI, donde sobresalen los vacunos, ovinos, porcinos, caprinos y aves (Flores, 2002).

En Huancavelica, la población actual de ganado vacuno es de 5156 cabezas, $14.7 \%$ mayor a la registrada en el censo agropecuario de 1994. La raza predominante es la criolla, representando el $63.9 \%$ del total de la distribución en el Perú (CENAGRO, 2012).

Según De Alva (1981), este bovino, adaptado a través del tiempo mediante una selección natural a las diferentes condicio- nes ambientales, ha adquirido rasgos característicos importantes como resistencia física, agilidad, adaptabilidad y resistencia a ectoparásitos (Rhipicephalus microplus) y enfermedades como anaplasmosis y babesiosis (Holgado, 1989). Además, es un animal que sobresale por su capacidad en el aprovechamiento de pastos naturales y mejorados, con una alta eficiencia reproductiva, longevidad, facilidad de las hembras para el parto y docilidad en el manejo (Bracho et al., 2002). Sin embargo, la producción de leche de estos animales es baja (450-1500 litros por campaña). La carne de las hembras es usualmente obtenida al final de su vida reproductiva, mientras que la de los machos ocuure luego de su labor como reproductores o unidades de tracción, caso contrario son vendidos a una edad más temprana para ser engordados y sacrificados (Flores, 2002).

El mal de altura es causa de pérdidas productivas y económicas. Holt y Callan (2007) mencionaron que las pérdidas por esta 
patología pueden abarcar el 3-5\% de animales en su primer año de vida. La hipoxia tiene un rol fundamental en la etiología del mal de altura, generando vasoconstricción pulmonar y aumento de la resistencia vascular, lo que se traduce en hipertensión pulmonar y posterior falla del lado derecho del corazón (Dunlop y Malbert, 2007; Malherbe et al., 2012). En este contexto, el ganado criollo tiene una mayor resistencia a esta patología que el ganado mejorado e introducido a las zonas altoandinas del Perú, lo cual hace suponer la existencia de genes ligados a la resistencia a la altura (Constable et al., 2016).

La variabilidad genética, base de las razas criollas, permitiría la obtención de líneas, especializadas en la producción de leche, carne o doble propósito, conservando sus características de adaptación y habilidad materna (Florio, 2008). Además, otra de las bondades que ofrece esta raza es la de asegurar un mayor vigor híbrido con los cruzamientos de individuos de otras razas, permitiendo terneros de media sangre con mayor peso al destete. Por tanto, el ganado criollo en el Perú es importante como pie de cría o base, sobre el cual se puede mejorar genéticamente, conservando sus características de rusticidad y adaptación a la altura (Flores, 2002).

Según Inchausti y Tagle (1982), la barimetría es aquella parte del estudio del exterior que permite estimar el peso vivo (PV) del animal mediante la aplicación de fórmulas basadas en medidas de diferentes regiones corporales, convirtiéndose en un instrumento útil en lugares donde no se pueden hacer uso de básculas. Menéndez (1984), considera que el aumento en el PV es el resultado de los cambios en la talla y forma de los animales; es decir, crecen en largo, ancho y altura. Por tanto, el desarrollo realmente medido o estimado, puede representar eficientemente el estado de crecimiento. Para evaluar el ganado bovino, se han venido utilizando entre seis a catorce medidas biométricas, siendo las más comunes la altura a la cadera, ancho de grupa, el perímetro torácico y la longitud corporal (Rodríguez et al., 2001; Abreu et al., 2005).

Matos (1978), Mahecha et al. (2002) y Khalil y Vaccaro (2002) analizaron las correlaciones fenotípicas entre diversas medidas biométricas y utilizaron regresiones simples o múltiples para obtener ecuaciones que predijeran el peso final de bovinos. En el Perú se dispone de escasa información sobre la interrelación de medidas corporales en ganado criollo. En base a esta razón el presente trabajo tiene como objetivo relacionar el PV con otras medidas biométricas y definir ecuaciones de predicción del PV en el grupo genético de bovinos criollos en Conayca, Huancavelica, Perú.

\section{MATERIALES y Métodos}

Se tomaron medidas biométricas a 90 bovinos criollos (Cuadro 1) entre agosto y setiembre de 2018 en la comunidad de Conayca, provincia de Huancavelica, ubicada a 3682 metros de altitud media. Las temperaturas promedio anual máxima en la zona es de $16{ }^{\circ} \mathrm{C}$ y la mínima de $2{ }^{\circ} \mathrm{C}$. La temporada seca comprende los meses de mayooctubre y la temporada lluviosa los meses de noviembre-abril, con una precipitación promedio anual de $784 \mathrm{~mm}$.

Cuadro 1. Distribución numérica de los bovinos criollos del estudio, según la edad dentaria y sexo (Conayca, Huancavelica, Perú)

\begin{tabular}{lccc}
\hline \multirow{2}{*}{$\begin{array}{l}\text { Edad } \\
\text { dentaria }\end{array}$} & \multicolumn{2}{c}{ Sexo } & \multirow{2}{*}{ Total } \\
\cline { 2 - 3 } & Macho & Hembra & \\
\hline $\begin{array}{l}\text { Dientes de } \\
\text { leche }\end{array}$ & 21 & 9 & 30 \\
2 dientes & 14 & 6 & 20 \\
4 dientes & 8 & 12 & 20 \\
Boca llena & - & 20 & 20 \\
\hline Total & 43 & 47 & 90 \\
\hline
\end{tabular}


La mayor parte de los animales fueron hembras (52.2\%). Los animales de dientes de leche participaron con el $33.3 \%$, mientras que el porcentaje de animales en las demás categorías fue de $22.2 \%$ (Cuadro 1). El sistema de crianza de los animales fue de tipo extensivo a base de pastizales constituidos principalmente de especies deseables (51.2\%): Alchemilla pinnata, Festuca dolichophylla y Disticha muscoides; poco deseables (42.0\%): Poa alquigluma, Calamagrostis ovata y Calamagrostis curvula, e indeseables (6.8\%): Aciachne pulvinata, Plantago rigida y Stipa obtusa (Quinto, 2004). Ocasionalmente algunos productores utilizaban heno de avena como alimentación suplementaria, y sal de piedra negra.

Teniendo en consideración las metodologías descritas por Rojas y Gomez (2005), se midieron las siguientes variables biométricas:

- Ancho de cabeza (AC): distancia entre los ángulos faciales de los ojos

- Longitud de cabeza (LC): distancia entre el testuz (parte media) y la punta del morro

- Ancho de tórax (ATO): distancia entre los puntos de encuentro derecho e izquierdo

- Altura de tórax (ALT): distancia de la cruz a la cinchera hasta el codo

- Longitud de cuerpo (LCU): distancia desde punto de encuentro hasta el isquion del mismo lado

- Perímetro torácico (PT): medida alrededor del tórax

- Altura a la cruz(ACR): distancia de base del piso a la cruz

- Ancho de grupa (AGR): distancia de punta de nalga-isquiones

- Longitud de grupa (LGR): distancie entre la tuberosidad iliaca al isquion

- Perímetro de caña anterior (PCA)

- Distancia dorso lumbar (DDL)

- Distancia de la cruz a la inserción de la cola

- Altura de grupa (ALG): distancia entre el piso y el nivel de la punta de anca
- Longitud de tórax (LTO): distancia desde la articulación del encuentro hasta mitad de la última costilla falsa

- Largo de cuerpo (LAC): distancia desde el testuz hasta la inserción de la cola

- Perímetro abdominal (PAB): medida alrededor del abdomen a nivel de la cicatriz umbilical

Las mediciones fueron realizadas por dos personas con apoyo de cuatro personas para la sujeción a nivel de cola, astas y verija en ambos flancos, en corrales de encierro acondicionados de malla y postes con instrumentos como bovinómetro de madera con graduación de 0 a $2 \mathrm{~m}$, hipómetro de madera, cinta métrica con graduación de 0 a $3 \mathrm{~m}$, compás de espesor de madera, graduado de $0 \mathrm{a}$ $0.55 \mathrm{~m}$.

El PV de los animales fueron estimados mediante la ecuación de predicción PV $=-321.04315+1.82146 \mathrm{PT}+0.78928 \mathrm{LAC}+$ 1.46924ACR $\left(\mathrm{R}^{2}=0.6143\right)$, descrita por Rojas et al. (2019), obtenido a partir de 96 bovinos criollos, procedentes del distrito de Huancavelica, Perú. Se determinaron correlaciones fenotípicas entre el peso corporal (PC) con las medidas biométricas: Ancho (AC) y longitud de cabeza (LC), ancho de tórax (ATO), ancho de grupa (AGR), longitud de grupa (LGR), distancia dorso lumbar (DL), altura de tórax (ALT), altura de grupa (AG), longitud de tórax (LT), perímetro torácico (PT), largo de cuerpo (LAC), longitud de cuerpo (LCU), altura de cruz (ACR) y perímetro abdominal (PA), para obtener una ecuación de predicción del PC de los bovinos criollos de Huancavelica. Los resultados fueron analizados mediante el análisis de variancia de clasificación múltiple para determinar el efecto del sexo y la edad dentaria de los animales en las medidas biométricas.

Se determinaron las correlaciones fenotípicas entre las medidas: PV, AC, LC, ATO, ALT, LCU, PT, ACR, AGR, LGR, PCA, DDL, ALG, LTO, LAC y PAB, en forma 
Cuadro 2. Resumen del análisis de variancia de las medidas biométricas en función al sexo y edad de 90 bovinos criollos (Conayca, Huancavelica, Perú)

\begin{tabular}{lcc}
\hline Fuente de variación & Sexo & Edad \\
\hline GL & 1 & 4 \\
Peso vivo (PV), kg & $\mathrm{ns}$ & $* * *$ \\
Ancho de cabeza (AC) & $\mathrm{ns}$ & $* * *$ \\
Longitud de cabeza (LC) & $\mathrm{ns}$ & $* * *$ \\
Ancho de tórax (ATO) & $\mathrm{ns}$ & $* *$ \\
Altura de tórax (ALT) & $\mathrm{ns}$ & $\mathrm{ns}$ \\
Longitud de cuerpo (LCU) & $\mathrm{ns}$ & $* * *$ \\
Perímetro torácico (PT) & $\mathrm{ns}$ & $* * *$ \\
Altura de cruz (ACR) & $\mathrm{ns}$ & $* * *$ \\
Ancho de grupa (AGR) & $\mathrm{ns}$ & $* * *$ \\
Longitud de grupa (LGR) & $\mathrm{ns}$ & $* * *$ \\
Perímetro de caña anterior (PCA) & $* * *$ & $* * *$ \\
Distancia dorso lumbar (DDL) & $\mathrm{ns}$ & $* * *$ \\
Altura de grupa (ALG) & $*$ & $* * *$ \\
Longitud de tórax (LTO) & $\mathrm{ns}$ & $* * *$ \\
Largo de cuerpo (LAC) & $\mathrm{ns}$ & $* * *$ \\
Perímetro abdominal (PAB) & $\mathrm{ns}$ & $* * *$ \\
\hline
\end{tabular}

ns: No significativo; ** $\mathrm{p}<0.01 ; * * *$ $\mathrm{p}<0.001$

conjunta de las observaciones (sexo y edades), recurriéndose al procedimiento CORR SPEARMAN del SAS (2009), v. 9.2. De acuerdo a Ribeiro et al. (2004) se consideraron las mayores correlaciones entre el PV y las características: LC, PT, ACR, ALG y LAC para ser sometidas a un análisis de regresión lineal múltiple de primer grado, a través del procedimiento REG del SAS (2009), con el siguiente modelo: $\mathrm{PV}_{\mathrm{ij}}=\mu+\mathrm{b}_{1} \mathrm{LC}+\mathrm{b}_{2} \mathrm{PT}+$ $\mathrm{b}_{3} \mathrm{ACR}+\mathrm{b}_{4} \mathrm{ALG}+\mathrm{b}_{5} \mathrm{LAC}+$ error (ij), donde $:$ PV = peso vivo de los bovinos criollos; $b_{1}, b_{2}, b_{3}, b_{4}$ y $b_{5}$ son los coeficientes de regresión de las características en estudio. Así mismo, fue sometido a análisis de regresión lineal múltiple de primer grado el PV con el PT y LAC por ser los mejores indicadores del PV (Khalil y Vaccaro, 2002; Ribeiro et $a l .$, 2004). Finalmente se generó una ecuación de regresión lineal entre el PV y el PT, conforme a lo descrito por los autores anteriores.

\section{Resultados y Discusión}

El sexo de los animales no fue fuente de variación significativa en las características evaluadas, excepto para el PCA $(p=0.0003)$ y ALG $(p=0.0294)$. La edad afectó de forma significativa en todas las características, excepto para ALT (Cuadro 2).

En la Cuadro 3 se presentan las medidas biométricas de los 90 animales criollos. La ALG y ACR muestran variabilidad baja, entre 8.72 y $8.93 \%$, y en concordancia con Cevallos et al. (2013). Las variabilidades para LC, ALT, LCU, PT, LGR, PCA, DDL, LAC y PAB fueron de tipo media (11.11-15.00\%), 
Cuadro 3. Medias, mínimos, máximos, desviaciones estándares y coeficientes de variabilidad de las características biométricas $(\mathrm{cm})$ de 90 bovinos criollos de Conayca, Huancavelica (Perú)

\begin{tabular}{lccccc}
\hline Características & Media & Mínimo & Máximo & $\begin{array}{c}\text { Desvío } \\
\text { estándar }\end{array}$ & $\begin{array}{c}\text { Coeficiente de } \\
\text { Variabilidad } \\
(\%)\end{array}$ \\
\hline Peso vivo (PV), kg & 224.20 & 58 & 348 & 58.36 & 26.03 \\
Ancho de cabeza (AC) & 20.81 & 12 & 35 & 4.58 & 22.01 \\
Longitud de cabeza (LC) & 42.76 & 29 & 55 & 4.75 & 11.11 \\
Ancho de tórax (ATO) & 27.88 & 16 & 40 & 5.48 & 19.66 \\
Altura de tórax (ALT) & 54.22 & 35 & 85 & 7.72 & 14.24 \\
Longitud de cuerpo (LCU) & 116.67 & 74 & 149 & 13.72 & 11.75 \\
Perímetro torácico (PT) & 147.57 & 98 & 184 & 17.36 & 11.76 \\
Altura de cruz (ACR) & 111.50 & 82 & 134 & 9.96 & 8.93 \\
Ancho de grupa (AGR) & 18.99 & 10 & 35 & 4.27 & 22.48 \\
Longitud de grupa (LGR) & 38.43 & 26 & 60 & 5.20 & 13.34 \\
Perímetro de caña anterior (PCA) & 16.11 & 12 & 20 & 2.07 & 12.84 \\
Distancia dorso lumbar (DDL) & 86.68 & 56 & 114 & 12.62 & 14.56 \\
Altura de grupa (ALG) & 115.19 & 89 & 136 & 10.05 & 8.72 \\
Longitud de tórax (LTO) & 70.98 & 41 & 163 & 18.80 & 26.49 \\
Largo de cuerpo (LAC) & 142.47 & 80 & 182 & 21.38 & 15.00 \\
Perímetro abdominal (PAB) & 162.30 & 100 & 196 & 22.87 & 14.09 \\
\hline
\end{tabular}

mientras que los coeficientes de variabilidad de las características AC, AGR, PV y LTO fueron altas, y de acuerdo con Cordero (2008). Estos resultados indican que existe una gran variación en la población, lo que es favorable para ser utilizado en programas de mejoramiento genético, pero debe destacarse que el PT $(184 \mathrm{~cm})$ y otros valores reportados del bovino criollo de la comunidad de Conayca se encontró una similitud con los estudios de More (2016) y Delgado et al. (2019).

En el Cuadro 4 se observan que las correlaciones entre el AC, ATO, LCU, AGR, LGR, PCA, DDL, LTO y PAB con el PV son positivas y moderadamente altas y significativas $(\mathrm{p}<0.001)$. Las características biométricas más correlacionadas con el PV fueron la LC, PT, ACR, ALG y LAC, con coeficientes, altos y significativos $(p<0.001)$
(Martínez et al., 2014), siendo estos coeficientes los más indicados para estimar el PV de los animales, en ambos sexos y con edades comprendidas entre dientes de leche y boca llena; sin embargo, todas las medidas relacionadas con el PV, excepto ALT, podrían ser utilizadas para expresar el PV de los bovinos criollos en estudio. Se observa que las correlaciones entre el PV y las medidas LC $=0.79, \mathrm{PT}=0.91, \mathrm{ACR}=0.77, \mathrm{ALG}=0.78 \mathrm{y}$ $\mathrm{LAC}=0.89$ son relativamente menores a lo relatado por Mahecha et al. (2002) en la raza Lucerna, quienes obtuvieron valores entre 0.94 y 0.97. Muñoz (2002) refiere que, si el tamaño de muestra es pequeño, el coeficiente de correlación (r) es una estimación ligeramente parcial y que subestima el coeficiente de correlación poblacional; hecho que probablemente haya ocurrido en el presente estudio, pues se trató de variables aleatorias bivariadas en número de 90 . 
Cuadro 4. Correlaciones fenotípicas de Spearman ${ }^{1}$ entre las características biométricas en bovinos criollos $(\mathrm{N}=90)$ de Conayca, Huancavelica (Perú), considerando el conjunto de animales (sexos y edades)

\begin{tabular}{|c|c|c|c|c|c|c|c|c|c|c|c|c|c|c|c|}
\hline & $\mathrm{PC}$ & $\mathrm{AC}$ & $\mathrm{LC}$ & ATO & ALT & LCU & PT & ACR & AGR & LGR & PCA & DDL & ALG & LTO & LAC \\
\hline $\mathrm{AC}$ & 0.57 & & & & & & & & & & & & & & \\
\hline LC & ${ }_{* * *}^{0.79}$ & ${ }_{* * *}^{0.59}$ & & & & & & & & & & & & & \\
\hline ATO & $\underset{* * *}{0.66}$ & $\underset{* * *}{0.65}$ & $\underset{* * *}{0.56}$ & & & & & & & & & & & & \\
\hline ALT & 0.44 & 0.32 & 0.27 & 0.42 & & & & & & & & & & & \\
\hline LCU & $\underset{* * *}{0.60}$ & $\underset{* * *}{0.48}$ & 0.56 & $\underset{* * *}{0.54}$ & $\underset{* *}{0.32}$ & & & & & & & & & & \\
\hline PT & 0.91 & $\underset{* * *}{0.48}$ & 0.79 & 0.55 & $0.26^{*}$ & 0.58 & & & & & & & & & \\
\hline ACR & $\underset{* * *}{0.77}$ & $\underset{* * *}{0.61}$ & 0.62 & $\underset{* * *}{0.53}$ & 0.50 & 0.42 & $\underset{* * *}{0.61}$ & & & & & & & & \\
\hline AGR & 0.58 & ${ }_{* * *}^{0.65}$ & $\underset{* * *}{0.65}$ & 0.52 & $0.26^{*}$ & 0.37 & 0.57 & 0.55 & & & & & & & \\
\hline LGR & 0.58 & $\underset{* * *}{0.35}$ & $\underset{* * *}{0.54}$ & 0.41 & ${ }_{* * *}^{0.50}$ & 0.52 & 0.56 & 0.54 & 0.40 & & & & & & \\
\hline PCA & 0.62 & 0.45 & 0.63 & 0.43 & $\begin{array}{c}0.17 \\
\text { ns }\end{array}$ & 0.41 & $\underset{* * *}{0.69}$ & $\underset{* * *}{0.48}$ & 0.62 & 0.35 & & & & & \\
\hline DDL & 0.67 & 0.72 & $\underset{* * *}{0.63}$ & $\underset{* * *}{0.49}$ & ${ }_{* * *}^{0.52}$ & 0.55 & $\underset{* * *}{0.52}$ & 0.79 & $\underset{* * *}{0.56}$ & $\underset{* * *}{0.46}$ & 0.44 & & & & \\
\hline ALG & 0.78 & ${ }_{* * *}^{0.50}$ & $\underset{* * *}{0.66}$ & $\underset{* * *}{0.59}$ & 0.34 & 0.69 & $\underset{* * *}{0.71}$ & 0.71 & 0.47 & 0.53 & 0.62 & 0.68 & & & \\
\hline LTO & ${ }_{* * *}^{0.67}$ & $\underset{* * *}{0.64}$ & $\underset{* * *}{0.60}$ & $\underset{* * *}{0.44}$ & $0.20^{*}$ & $\underset{* * *}{0.67}$ & $\underset{* * *}{0.64}$ & $\underset{* * *}{0.59}$ & $\underset{* * *}{0.52}$ & $\underset{* * *}{0.39}$ & ${ }_{* * *}^{0.57}$ & $\underset{* * *}{0.65}$ & $\underset{* * *}{0.64}$ & & \\
\hline LAC & $\underset{* * *}{0.89}$ & $\underset{* * *}{0.51}$ & $\underset{* * *}{0.63}$ & ${ }_{* * *}^{0.68}$ & $\underset{* * *}{0.48}$ & 0.58 & $\underset{* * *}{0.72}$ & 0.65 & 0.49 & 0.48 & ${ }_{* * *}^{0.53}$ & ${ }_{* * *}^{0.65}$ & $\underset{* * *}{0.73}$ & ${ }_{* * *}^{0.55}$ & \\
\hline PAB & ${ }_{* * *}^{0.66}$ & $\underset{* *}{0.32}$ & $\underset{* * *}{0.65}$ & $\underset{* *}{0.33}$ & $\begin{array}{c}0.07 \\
\mathrm{~ns}\end{array}$ & 0.42 & $\underset{* * *}{0.72}$ & $\underset{* * *}{0.47}$ & $\underset{* * *}{0.54}$ & $\underset{* *}{0.29}$ & ${ }_{* * *}^{0.66}$ & $\underset{* * *}{0.45}$ & $\underset{* * *}{0.73}$ & $\begin{array}{l}0.54 \\
* * *\end{array}$ & ${ }_{* * *}^{0.55}$ \\
\hline
\end{tabular}

${ }^{1} \mathrm{PV}=$ peso vivo; $\mathrm{AC}=$ ancho de cabeza; $\mathrm{LC}=$ longitud de cabeza; $\mathrm{ATO}=$ ancho de tórax; $\mathrm{ALT}=$ altura de tórax; $\mathrm{LCU}=$ longitud de cuerpo; $\mathrm{PT}=$ perímetro torácico; $\mathrm{ACR}=$ altura de cruz; $\mathrm{AGR}$ = ancho de grupa; $\mathrm{LGR}=$ longitud de grupa; $P C A$ = perímetro de caña anterior; $D D L$ = distancia dorso lumbar; $A L G$ = altura de grupa; LTO = longitud de tórax; $\mathrm{LAC}=$ largo de cuerpo; $\mathrm{PAB}=$ perímetro abdominal ns No significativo; $* * p<0.01 ; * * * p<0.001$

Entre las correlaciones anteriores, el PT y LAC presentaron los mayores coeficientes de correlación con el PV (0.91 vs. 0.89). El PT, según la literatura científica, es la característica más correlacionada con el PV. Así, se establecieron correlaciones de 0.90 en ganado Pardo Suizo (Henao y Mejía, 1994), 0.98 en ganado cebú (Méndez et al., 2002), 0.93 en terneros machos cebú (Hernández et al., 1984), 0.90 y 0.93 en ganado bovino mestizo venezolano y en la raza Carora (Khalil y Vaccaro, 2002).

El análisis de variancia de la regresión evidenció la influencia significativa $(\mathrm{p}<0.01)$ de las características en el PV de los bovinos criollos en cada una de las ecuaciones generadas (Cuadro 5). La ecuación de predicción $\mathrm{PV}=-321.11+1.82 * * * \mathrm{PT}+1.47 * * * \mathrm{ACR}+$ $0.79 * * *$ LAC $\left(\mathrm{R}^{2}=1.0000\right)$ muestra que exis- 
Cuadro 5. Ecuaciones de predicción del peso vivo (PV) en función al perímetro torácico (PT), altura de cruz (ACR) y largo de cuerpo (LAC)

\begin{tabular}{llc}
\hline Características & \multicolumn{1}{c}{ Ecuación } & $\mathrm{R}^{2}$ \\
\hline $\mathrm{PT}+\mathrm{ACR}+\mathrm{LAC}$ & $\mathrm{PV}=-321.11+1.82^{* * *} \mathrm{PT}+1.47^{* * *} \mathrm{ACR}+0.79^{* * *} \mathrm{LAC}(\mathrm{I})$ & 1.0000 \\
$\mathrm{PT}+\mathrm{LAC}$ & $\mathrm{PV}=-255.05+2.32^{* * *} \mathrm{PT}+0.96^{* * *} \mathrm{LAC}(\mathrm{II})$ & 0.9775 \\
$\mathrm{PT}$ & $\mathrm{PV}=-253.41+3.24^{* * *} \mathrm{PT}(\mathrm{III})$ & 0.9279 \\
\hline${ }^{* * *} \mathrm{p}<0.001$ & &
\end{tabular}

te una asociación positiva entre las características, y que cada centímetro del PT, de la ACR y del LAC presentan una contribución positiva de $1.82,1.47$ y $0.79 \mathrm{~kg}$ en el PV de los bovinos criollos en estudio; mientras que las medidas LC y ALG consideradas en el modelo original no aportaron con respecto a la predicción del PV de los bovinos criollos en estudio.

De acuerdo con la selección de las variables, en los modelos I y II de predicción, el PT ocupa la primera posición, seguido por la ACR y el LAC. Mahecha et al. (2002), por otra parte, en la raza Lucerna indican que el orden de prioridad de las mediciones corporales con relación a los mayores coeficientes de correlación fueron el perímetro torácico $>$ altura corporal $>$ longitud corporal en el casio de las hembras, y el perímetro torácico $>$ altura corporal $>$ longitud corporal en el caso de los machos; comportamiento que está en concordancia con la posición del PT en las ecuaciones obtenidas al utilizar animales de diferentes edades y de sexo en el presente estudio.

Aplicando valores ficticios de $158 \mathrm{~cm}$ para PT, $152 \mathrm{~cm}$ para LAC y $120 \mathrm{~cm}$ para ACR, los pesos fueron de 258.51 y 257.43 $\mathrm{kg}$ cuando se estimaron a partir de PT solo (Ecuación III) y cuando se adicionó LAC (Ecuación II), respectivamente; es decir, se estimaron pesos similares. Así mismo, cuando se adicionó ACR al PT y al LAC (Ecuación I) el PV estimado fue de $263.04 \mathrm{~kg}$, superando en 5.61 y $4.61 \mathrm{~kg}$ los pesos obteni- dos con las ecuaciones II y III, respectivamente.

\section{Conclusiones}

- Existe una amplia variación en el peso vivo, ancho de cabeza, ancho de grupa y longitud de tórax, y en menor grado, en la altura de cruz y altura de grupa en bovinos criollos de la zona de Huancavelica, Perú.

- Las características perímetro torácico, largo de cuerpo, altura de cruz y altura de grupa fueron las medidas con mejor correlación con el peso vivo y, consecuentemente, las más apropiadas para estimar el peso de los animales.

- Las ecuaciones generadas a partir del perímetro torácico solo o adicionadas del largo de cuerpo ofrece a los ganaderos criadores de bovino criollo obtener pesos similares. La adición de la altura de cruz a la ecuación de predicción mejora la precisión en la obtención de los pesos.

\section{Agradecimiento}

A los fondos económicos concursables de Fondo de Desarrollo Socioeconómico del Proyecto Camisea por el financiamiento a través del proyecto «Generación y evaluación de un sistema computarizado en la formulación de raciones al mínimo costo en ganado lechero en la provincia de Huaytará, 
Acobamba, Tayacaja y Huancavelica de la Región Huancavelica». Así mismo los autores agradecen el apoyo de la estudiante Analí Ñaupa Ccente (Q.D.E.P.).

\section{Literatura Citada}

1. Abreu UGP de, Santos SA, Sereno JRB, Comastri-Filho MS, Ravanelli MS. 2005. Caracterización morfo métrica de los bovinos Pantaneiros del núcleo de conservación in situ de Nhumirin. Arch Zootec 54:211-216.

2. Bracho I, Contreras M, Pirela M, Zambrano S. 2002. La raza Criollo Limonero: una realidad para la ganadería de doble propósito. En: Gonzales-Stagnaro C, Soto Belloso E, Ramírez Iglesia L (eds). Avances en la ganadería de doble propósito. Maracaibo, Venezuela: Astro Data. p 9-25.

3. Caballero $W$. 2006. Pobreza y pobreza extrema rural: en la pequeña agricultura y en la agricultura de minifundio. Lima, Perú: Univ. Nacional Agriaria La Molina. 454 p.

4. CENAGRO. 2012. IV Censo Nacional Agropecuario. [Internet]. Disponible en: https://www.agrorural.gob.pe/ dmdocuments/resultados.pdf

5. Cevallos FO, Estupiñán VK, Torres $\mathrm{Y}$, Garcia A. 2013. Caracterización morfoestructural y faneróptica del bovino Criollo de la provincia de Manabí, Ecuador. En: XXII Reunión de la Asociación Latinoamericana de Producción Animal- ALPA. La Habana, Cuba.

6. Constable PD, Hinchcliff $K W$, Done SH, Grünberg $W$. 2016. Veterinary medicine: a textbook of the diseases of cattle, horses, sheep, pigs and goats. $11^{\text {th }}$ ed. Elsevier. 2278 p.

7. Cordero FA. 2008. Estadística experimental. Soluciones con los aplicativos SAS, SPSS y EXCEL en experimentos zootécnicos y agronómicos. Huancayo, Perú: Grapex Perú. 324 p.

8. De Alba J. 1981. Recursos genéticos animales en América Latina. Ganado criollo y especies de altura. Roma, Italia: FAO. 168 p.
9. Delgado A, García C, Allcahuamán D, Aguilar C,Estrada P, Vega H. 2019. Caracterización fenotípica del ganado criollo en el Parque Nacional Huascarán Ancash, Perú. Rev Inv Vet Perú 30: 11431149. doi: 10.15381/rivep.v30i3.16611

10. Dunlop R, Malbert C. 2007. Fisiopatología veterinaria. España: Acribia. $556 \mathrm{p}$.

11. Flores $M A$. 2002. Visión panorámica de la ganadería. En: Caballero W, Flores A, Arroyo O, Alcántara A (eds). Hacia una nueva agricultura, con énfasis en la generación y transferencia de tecnología. Lima: CONCYTEC. p 245-314.

12. Florio J. 2008. Uso de los bovinos criollos en cruzamientos con otras razas bovinas en América Latina, con énfasis en ganadería doble propósito. En: Desarrollo sostenible de la ganadería de doble propósito. Venezuela. [Internet]. Disponible en: http://www.avpa.ula.ve/ libro_desarrollosost/pdf/capitulo_10.pdf

13. Henao F, Mejía N. 1994. Barimetría en ganado Pardo Suizo. Rev Vet Zoot Caldas 7(4): 78-81.

14. Hernández M, García F, Sotto V, López M. 1984. Estimado del peso vivo al nacer en terneros cebú. Asoc Cubana Prod Anim 3: 42.

15. Holgado F. 1989. El bovino criollo en la República Argentina. En: Diálogo XXXVI. Conservación y mejoramiento del ganado bovino Criollo. IICA.PROCISUR. p 25-34.

16. Holt T, Callan R. 2007. Pulmonary arterial pressure testing for high mountain disease in cattle. Vet Clin N Am-Food A 23: 575-596. doi: 10.1016/j.cvfa.2007.08.001

17. Inchausti D, Tagle CT. 1982. Bovinocultura: exterior y razas. Buenos Aires: El Ateneo. $230 \mathrm{p}$

18. Khalil R, Vaccaro L. 2002. Peso y mediciones corporales en vacas de doble propósito: su interrelación y asociación con valor genético para tres características productivas. Zootec Trop 20:11-30.

19. Mahecha L, Angulo J, Manrique LP. 2002. Estudio bovinométrico y relaciones entre medidas corporales y el peso 
vivo en la raza Lucerna. Rev Colomb Cienc Pec 15: 80-87.

20. Malherbe C, Marquard J, Legg D, Cammack K, O'Toole D. 2012. Right ventricular hypertrophy with heart failure in Holstein heifers at elevation of 1600 meters. J Vet Diagn Invest 24: 867-877. doi: $10.1177 / 1040638712453580$

21. Martínez LOR, Núñez L, Castro L, Rodríguez MI, Álvarez R, Florentín A, Ramírez L, et al. 2014. Uso de correlación estadística para el estudio morfométrico de bovinos de carne: Caso Pampa Chaqueño. Comp Cien Vet 4: 26-32.

22. Matos RR. 1978. Correlações entre medidas morfológicas e estimativas do desempenho de bovinos da raça Hereford submetidos a teste de avaliação. En: XV Reunião Anual da Sociedad Brasileira de Zootecnia. Belem, Pará.

23. Méndez MM, Serrano PJ, Avila BR, Rosas GM, Méndez PN. 2002. Características morfométricas del bovino criollo Mixteco. Arch Zootec 51: 217-221.

24. Menéndez B. 1984. Un método simple para evaluar hembras lecheras. Asoc Cubana Prod Anim 3:13-19.

25. More M. 2016. Caracterización faneróptica y morfométrica del vacuno criollo en Ayacucho, Puno y Cajamarca. Te- sis de Maestría. Lima: Univ. Nacional Agraria La Molina. $65 \mathrm{p}$.

26. Muñoz SA. 2002. Estadística aplicada uni y multivariante. Tomo I y II. España: Universidad de Córdoba.1018 p.

27. Rodríguez MG, Fernández G, Silveira C, Delgado JV. 2001. Estudio étnico de los bovinos Criollos del Uruguay. Arch Zootec 50: 113-118.

28. Rojas E, Gomez U. 2005. Biometría y constantes clínicas del bovino criollo en el centro de investigación y producción de Chuquibambilla de Puno (Perú). Arch Zootec 54: 233-236.

29. Rojas Y, Contreras J, Del Solar J, Repuello W, Ramos C. 2019. Predicción del peso vivo en función a medidas morfometricas en bovinos criollos en la Región Huancavelica. En: XLII Reunión Científica Anual de la Asociación Peruana de Producción Animal -APPA. Tingo María, Perú.

30. Ribeiro MM, Da Silva JV, Pimenta FEC, Sereno JRB. 2004. Estudio de las correlaciones entre características fenotípicas de caprinos naturalizados. Arch Zootec 53: 337-340.

31. SAS. 2009. SAS/STAT: user's Guide: Statistics. Release 9.2. SAS Institute Inc. Cary, NC. 